\title{
Case Report: SPECT/CT as the New Diagnostic Tool for Specific Wrist Pathology
}

\section{Linde Musters ${ }^{1^{\star}}$, Ten Broek $\mathbf{M}^{2}$ and Kraan GA ${ }^{1}$}

${ }^{1}$ Department of Orthopaedic Surgery, Reinier de Graaf Hospital Delft, The Netherlands

${ }^{2}$ Department of Nuclear Imaging, Reinier de Graaf Hospital, Delft, The Netherlands

"Corresponding author: Linde Musters, MD, Department of Orthopaedic Surgery, Reinier de Graaf Hospital, Delft, Mathenesserdijk 266a1, 3026GN, Rotterdam, the Netherlands, Tel: +31644996221; E-mail: lindemusters@gmail.com

Received date: July 12, 2017; Accepted date: July 17, 2017; Published date: July 21, 2017

Copyright: $\odot 2017$ Musters L, et al. This is an open-access article distributed under the terms of the Creative Commons Attribution License, which permits unrestricted use, distribution, and reproduction in any medium, provided the original author and source are credited.

\section{Abstract}

Introduction: Single photon emission computed tomography has been introduced as a promising new diagnostic tool in orthopaedic pathology since the early 90'. Computed tomography, the combined with SPECT, gives insight in the specific sight of wrist pathology. Literature already supports introduction of SPECT/CT in wrist pathology, but clinical application is lagging.

Case Report: A 40yr old patient reported first in 2004 with persisting pain after a right distal radius fracture. Several diagnostics and operative interventions were performed, all unsuccessful. Because of the persisting pain a SPECT-CT was performed which showed a cyst in the hamate bone, which was successfully enucleated. The patient was finally pain free at recent follow-up. With a QDash-score of 43 and a PRW (H) E-DLV-score of 58/150.

Discussion: In this case report, SPECT/CT proved a very sensitive diagnostic tool for specific pathology of the wrist. It offered precise localisation and thereby the clinically suspected diagnosis was confirmed and the patient successfully treated.
\end{abstract}

Keywords: Proximal row carpectomy; Single-Photon EmissionComputed Tomography; SPECT/CT; Capitate; Hamate; Wrist

Abbreviations SPECT/CT: Single Photon Emission Computed Tomography; mCi: Millicurie; CT: Computed Tomography; mSV: Milli Sievert; MRI: Magnetic Resonance Imaging; PRC: Proximal Row Carpectomy

\section{Introduction}

Single photon emission computed tomography (SPECT) has been introduced as a promising new diagnostic tool in orthopaedic pathology since the early 90's [1-3]. Dubowitz et al. [4] already concluded that combining scintigraphy and SPECT improved the localization of abnormalities in the wrist, but to distinguish between the carpal bones remained difficult [4].

The need for imaging that would give insight in the exact pathological sight raised. Hirschmann et al. [5] described a new technique of embedding computed tomography, the precise anatomic localization and SPECT, abnormal bone metabolism [4-6].

Could this new technique be able to offer insight in the exact localization of the pathology (Figure 1) in uncomprehend wrist pathology? SPECT/CT is a technique where computed tomography (CT) scan is combined with metabolism and a 3-dimensional image $[7,8]$. Radiation exposure is only $2-5 \mathrm{mSV}$ [9]. This technique has already been applied into several clinical orthopaedic pictures; femoro acetabular impingement, meniscal tears, ligament injuries, osteochondral lesions, osteochondritis dissecans etc. [5].

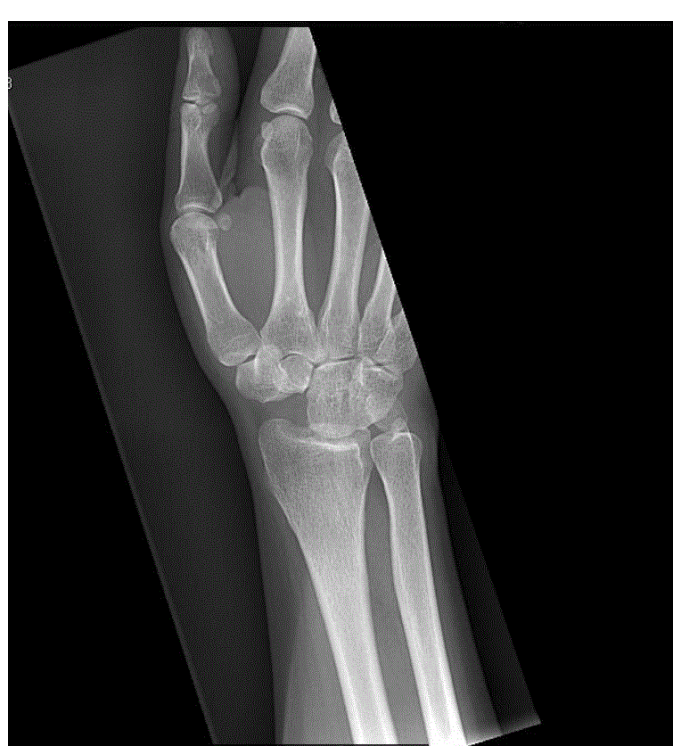

Figure 1: X-ray of 2011 of the right wrist after proximal row carpectomy.

We want to present a case in which the SPECT/CT was very helpful (Figure 2). 


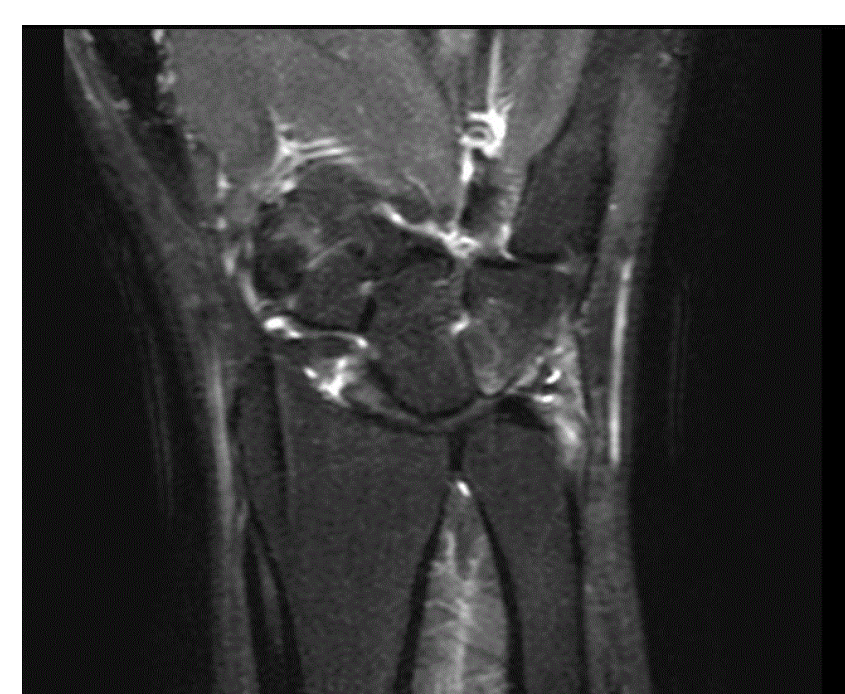

Figure 2: MRI of $2011 \mathrm{~T} 2$ of the right wrist showing synovitis at the trapezial radial joint.

\section{Case Report}

A right hand dominant $40 \mathrm{yr}$ old woman reported to the hospital in June 2004 with persisting pain after a right distal radius fracture. An arthroscopy shows slight synovitis of the wrist, laxity of the SL ligament and avascular necrosis of the proximal pool of the scaphoid bone. 6 Months later pain is progressing and function is decreased with 50 percent. CT-scan showed a scapholunate advanced collapse (SLAC) type III osteoarthritic wrist for which an uncomplicated proximal row carpectomy (PRC) was performed.

5 years after the operation patient returned with increasing pain of the right wrist mainly at the radial trapezium transition. MRI showed osteoarthritis at the radiocarpal transition and edema of the hamate bone and a tear in the triangular fibrocartilage complex, not responding to physiotherapy or kenacort injections. Because of persisting pain a bone scintigraphy was performed, which showed a cyst of the hamate bone, followed by arthroscopic debridement.

Because of still persisting pain a SPECT-CT was performed which showed a cyst in the hamate bone at the articulation. 3 months later thee cyst was enucleated and an arthrodesis (Figure 3) of capitate and hamate bone was performed. Control X-ray of the wrist showed a fusion of the proximal pole of the hamate and capitate interval. Radiographic control showed an increasing fusion, which is being follow-up through the outpatient clinic and improved with continuing hand therapy. With the QDash-score 43 and the PRW (H) E-DLVscore 58/150. The palmar dorsal flexion was 40-50 degrees.

\section{Discussion}

In this case report, SPECT/CT proved a very helpful diagnostic tool for pathology of the wrist. Several diagnostic and operative interventions during 8 years, for example; $\mathrm{X}$ ray, CT scanning, MR Imaging and even an arthroscopy could not identify the specific pathology and successfully treat the pain.

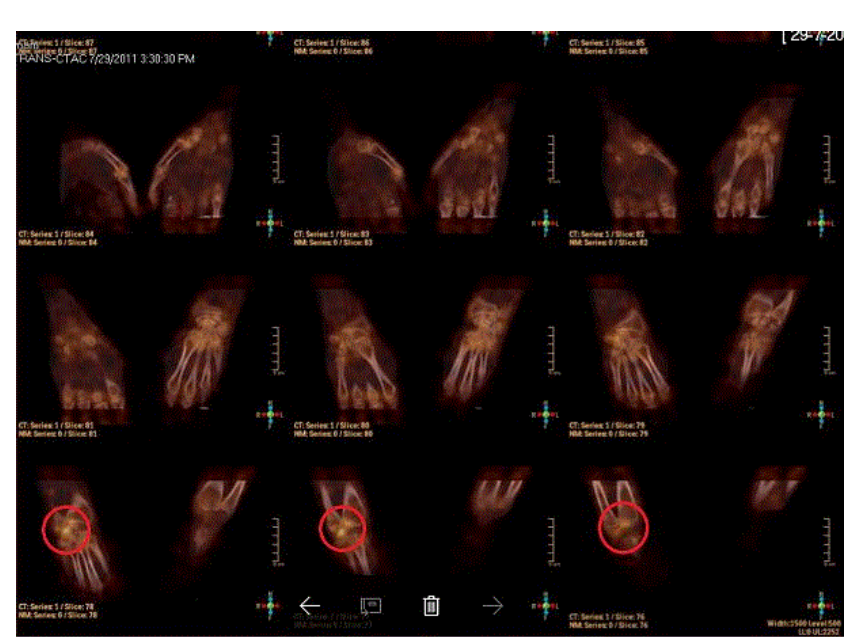

Figure 3: SPECT/CT of 2011 of the right wrist showing high signal at the hamate bone, shaded with the red circles.

Disadvantages of SPECT/CT are the longer duration, to blame to the time needed to collect enough fotons. Patients have to lay very still, small movements can lead to disturbance of the images.

SPECT/CT eventually combined bone activity with localisation of the anatomical area on CT and treatment of the shown pathology was successful.

In a study by Hirschmann and co-workers, the value of SPECT/CT was demonstrated in patients with pain after surgical treatment of knee osteoarthritis [6]. They found that the SPECT/CT showed the highest value in radiological support of clinical suspected diagnoses by displaying the specific anatomical areas of the shown pathology. Furthermore diagnostic sensitivity of SPECT/CT when used to differentiate between benign and malignant bone lesions is $98.4 \%$ compared to plain SPECT with $82.5 \%$ [10,11]. A study by Stolfuss et al. showed that SPECT had a higher sensitivity for chronic osteoarthritis in foot and ankle than planar imaging ( 0.80 vs. 0.68 , n.s.).

The advantage of SPECT was most obvious in the anatomically complex midfoot area (0.63 vs. $0.26, \mathrm{p}<0.05)$ [12]. The value of SPECT/CT in specific pathology of the joints has been demonstrated in several other studies as well $[3,5,6]$. The current treatment by a PRC shows many patients with post-operative complaints of pain, who required daily medication and were unable to return to manual labor type jobs. Hopefully further research on the employability of SPECT/CT for chronic complaint could have some benificial effect for this population.

In this case report we could confirm the above mentioned results that SPECT/CT offers more precise localisation and thereby, the clinically suspected diagnosis was confirmed and treated successfully.

\section{Authors' Contributions}

L. Musters; conception and design, provision of patient, collection/ assembly of data, data analysis/interpretation, literature search, manuscript writing.

G.A. Kraan; revising and final approval.

M. ten Broek; data analysis/interpretation 
Citation: Musters L, Broek TM, Kraan GA (2017) Case Report: SPECT/CT as the New Diagnostic Tool for Specific Wrist Pathology. J Arthritis 6:

Page 3 of 3

\section{Consent}

Did the author obtain written informed consent from the patient for submission of this manuscript for publication? Yes.

\section{Human and Animal Rights}

Ethical standards followed the responsible committee on human experimentation (institutional and national) and with the Helsinki Declaration of 1975, as revised in 2000.

\section{Acknowledgments}

Jasmijn W Selten, Department of Surgery, Erasmus MC-University Medical Centre, Rotterdam, Netherlands and W. Bramer, Biomedical Information specialist, Erasmus MC-University Medical Centre, Rotterdam, Netherlands. This research work was supported by funding from NIH Grant Number: 1R21NS082546-01A1.

\section{References}

1. Beyer T, Freudenberg LS, Townsend DW, Czernin J (2011) The future of hybrid imaging-part 1: hybrid imaging technologies and SPECT/CT. Insights Imaging 2: 161-169.

2. Buch K, Blumenfeld H, Spencer S, Novotny E, Zubal IG (2008) Evaluating the accuracy of perfusion/metabolism (SPET/PET) ratio in seizure localization. Eur J Nucl Med Mol Imaging 35: 579-588.

3. Schleich FS, Schürch M, Huellner MW, Hug U, Wartburg UV, et al. (2012) Diagnostic and therapeutic impact of SPECT/CT in patients with unspecific pain of the hand and wrist. EJNMMI Res 2: 53.

4. Dubowitz DJ, Miles KA (1994) Technical note: bone SPECT of the wrist. Br J Radiol 67: 890-893.
5. Hirschmann MT, Davda K, Rasch H, Arnold MP, Friederich NF (2011) Clinical value of combined single photon emission computerized tomography and conventional computer tomography (SPECT/CT) in sports medicine. Sports Med Arthrosc 19: 174-181.

6. Hirschmann MT, Iranpour F, Davda K, Rasch H, Hügli R, et al. (2010) Combined single-photon emission computerized tomography and conventional computerized tomography (SPECT/CT): clinical value for the knee surgeons? Knee Surg Sports Traumatol Arthrosc 18: 341-345.

7. Hirschmann MT, Iranpour F, Konala P, Kerner A, Rasch H, et al. (2010) A novel standardized algorithm for evaluating patients with painful total knee arthroplasty using combined single photon emission tomography and conventional computerized tomography (SPECT/CT). Knee Surg Sports Traumatol Arthrosc 18: 939-944.

8. Pagenstert GI, Barg A, Leumann AG, Rasch H, Müller-Brand J, et al. (2009) SPECT-CT imaging in degenerative joint disease of the foot and ankle. J Bone Joint Surg Br 91: 1191-1196.

9. Henckel J, Richards R, Lozhkin K, Harris S, Rodriguez y Baena FM, et al. (2006) Very low-dose computed tomography for planning and outcome measurement in knee replacement. The imperial knee protocol. J Bone Joint Surg Br 88: 1513-1518.

10. Ali MH, Rizzo M, Shin AY, Moran SL (2012) Long-term oudcomes of proximal row carpectomy; a minimum of 15 -year follow-up. Hand (N Y) 7: $72-78$.

11. Zhao Z, Li FL, Zhao LX, Su MG (2008) SPECT/CT fusion imaging for the diagnosis of benign and malignant lesions in bones. Sichuan Da Xue Xue Bao Yi Xue Ban 39: 1036-1039.

12. Stollfuss JC, Freudenberg LS, Wieder H (2016) 99mTc-DPD SPECT/CT for localisation of inflammatory and chronic osteoarthritis of the foot and ankle. Comparison with planar imaging and SPECT as a clinical adjunct in radiosynovectomy. Nuklearmedizin 55: 145-150. 